\title{
Upregulation of the alternative splicing factor NOVA2 in colorectal cancer vasculature
}

This article was published in the following Dove Press journal: OncoTargets and Therapy

\author{
Stefania Gallo',* \\ Maria Vittoria \\ Arcidiacono ${ }^{2, *}$ \\ Veronica Tisato' \\ Roberta Piva ${ }^{3}$ \\ Letizia Penolazzi ${ }^{3}$ \\ Cristina Bosi' \\ Carlo V Feo' \\ Roberta Gafà' \\ Paola Secchiero' \\ 'Department of Morphology, \\ Surgery, Experimental Medicine and \\ LTTA Center, University of Ferrara, \\ Ferrara, Italy; ${ }^{2}$ Institute for Maternal \\ and Child Health, IRCCS "Burlo \\ Garofolo," Trieste, Italy; ${ }^{3}$ Department \\ of Biomedical and Specialty Surgical \\ Sciences, University of Ferrara, \\ Ferrara, Italy \\ *These authors contributed equally \\ to this work
}

Background: Tumor-specific isoforms generated by alternative splicing (AS) are demonstrated to contribute to tumor progression and can represent potential biomarkers. NOVA2 is an AS factor that in physiological conditions regulates endothelial cells' (ECs) polarity and vessel lumen maturation, likely by mediating AS of apical-basal polarity regulators. However, NOVA2 expression in tumor ECs and its regulation have never been investigated.

Methods: To elucidate this, 40 colorectal cancer patients were enrolled and NOVA2 expression was investigated by immunohistochemistry in samples bearing both the normal mucosa and the tumor tissue.

Results: NOVA2 was found expressed in ECs of tumor vasculature and, importantly, it was upregulated in tumor ECs with respect to normal mucosa ECs in all cases $(P<0.001)$. The same samples analyzed by immunohistochemistry for the expression HIF1 $\alpha$, a marker of hypoxia, showed a positive and significant association with NOVA2 levels $(P=0.045)$. Of note, NOVA2 was upregulated by hypoxia also in an in vitro ECs model.

Conclusion: Our results provide, for the first time, evidence of NOVA2 expression and upregulation in tumor ECs and highlight hypoxia as a potential regulatory factor. These findings open a completely new perspective to study tumor vasculature and to uncover NOVA2 as a potential source of biomarkers and therapeutic targets based on AS isoforms.

Keywords: NOVA2, tumor angiogenesis, colorectal cancer, hypoxia

\section{Introduction}

Alternative splicing (AS) is the mechanism that generates multiple mRNAs from the same gene, leading to proteomic and functional diversity, and it is mediated by AS factors that, by recognizing RNA sequences on immature transcripts, facilitate the inclusion or exclusion of exons and introns. ${ }^{1}$ NOVA 1 and NOVA2 proteins are AS factors initially described to be expressed in a mutually exclusive manner in different central nervous system territories where they regulate AS of genes involved in synaptogenesis and neuron development. ${ }^{2}$ In addition, NOVA2 expression has been recently revealed in endothelial cells (ECs), adipocytes, and pancreatic beta cells as well. ${ }^{3-6}$ In particular, it has been demonstrated that in normal endothelium NOVA2 is essential for the establishment of ECs polarity and lumen maturation. ${ }^{4}$ In a previous study, Giampietro et $\mathrm{al},{ }^{4}$ using human and murine in vitro endothelial models and an in vivo zebrafish model, demonstrated for the first time that NOVA2 mediates the AS of apical-basal polarity regulators and that NOVA2 depletion resulted in impaired ECs permeability, directional cell migration, and junction architecture. While NOVA1, which is highly related to NOVA2, has been described to be expressed in different tumor types, ${ }^{7}$ no evidence has been reported about NOVA2 expression in human tumors.
Correspondence: Stefania Gallo Department of Morphology, Surgery, Experimental Medicine and LTTA

Center, University of Ferrara, via Fossato di Mortara 70, Ferrara 44I2I, Italy

Tel +39053245584l

Email stefania.gallo@unife.it (c) (1) (5) 2018 Gallo et al. This work is published and licensed by Dove Medical Press Limited. The full terms of this license are available at https://www.dovepress.com/terms.php (c) hereby accept the Terms. Non-commercial uses of the work are permitted without any further permission from Dove Medical Press Limited, provided the work is properly attributed. For permission for commercial use of this work, please see paragraphs 4.2 and 5 of our Terms (https://www.dovepress.com/terms.php). 
During tumor angiogenesis, the formation of new vessels allows oxygen and nutrient delivery to tumor cells, and it is mostly promoted by hypoxia through the induction of hundreds of genes. ${ }^{8-11}$ Notably, there is a growing interest in studying tumor angiogenesis, not only to overcome the flaws of current antiangiogenic therapies but also to identify and characterize new tumor-specific endothelial biomarkers for the development of novel therapeutic molecules.

The association of aberrant AS to tumor growth has been described in many cancer types and has been proposed as potential tumor biomarker. ${ }^{12}$ Nevertheless, NOVA2 expression in tumor vasculature has never been explored. Here, we have investigated NOVA2 expression in a cohort of colorectal cancer (CRC) patients and studied its association with hypoxia.

\section{Materials and methods}

\section{Patients and samples}

Tissue specimens were collected after obtaining written informed consent permission from $40 \mathrm{CRC}$ patients undergoing curative resection at the University Hospital S. Anna of Ferrara over an 18-month period. Twenty-three patients $(57.5 \%)$ were male, and 17 (42.5\%) were female; the average age was $71 \pm 13$ years. None of the patients underwent anticancer therapies before surgery. Twenty-five tumors $(62.5 \%)$ were located in the right colon, $9(22.5 \%)$ in the left colon and $6(15 \%)$ in the rectum. Eight tumors (20\%) were classified as mucinous adenocarcinoma and $32(80 \%)$ as conventional adenocarcinoma. Thirty-three tumors $(82.5 \%)$ were classified as low grade and $7(18 \%)$ as high grade. Tumors were staged according to the TNM eighth edition by American Joint Committee on Cancer: $6(15 \%)$ stage I, 21 (52.5\%) stage II, 7 (17.5\%) stage III, and 6 (15\%) stage IV. Twenty-seven tumors (67.5\%) were pN0, 10 (25\%) were $\mathrm{pN} 1$, and $3(7.5 \%)$ were $\mathrm{pN} 2$. Thirty-four tumors (85\%) were pM0 and 6 (15\%) were pM1.

Seven out of 40 CRC cases (17.5\%) showed high microsatellite instability (MSI-H) and presented loss of expression of at least one of the mismatch repair (MMR) proteins by immunohistochemistry, while the remaining cases $(82.5 \%)$ were microsatellite stable (MSS) without presenting loss of expression of MMR proteins. MMR proteins status was determined by immunohistochemistry using Ventana EnVision method and a 4-antibody panel of MMR proteins including MLH1, MSH2, MSH6, and, in some cases, PMS2; all the antibodies were ready-to-use monoclonal antibodies (MLH1 clone M1; MSH2 clone G219-1129; MSH6 clone 44; PMS2 clone EPR3947, Roche Diagnostics, Risch-Rotkreuz, Switzerland). Tumors demonstrating absolute absence of nuclear staining were classified as negative for protein expression, provided internal controls were positive. To test the MSI status, 4 microsatellite loci (BAT25, BAT26, BAT40, D5S346) were evaluated using a fluorescence-based PCR method. According to the guidelines of the International Workshop of Bethesda, tumors showing instability at $\geq 30 \%$ of microsatellite loci were classified as MSI-H and tumors without detectable MSI were classified as MSS.

An additional patient with rectal cancer (staged as ypT2-N0) undergoing neoadjuvant chemo/radiotherapy was enrolled for a preliminary evaluation of the effect of therapy on NOVA2 expression.

\section{Immunohistochemistry}

Tumor sections stained with haematoxylin and eosin were reviewed by two experienced gastrointestinal pathologists for histopathological analysis. For each case, a representative single formalin-fixed paraffin-embedded tissue block including both the tumor tissue and the adjacent normal mucosa was selected. Four micrometer sections were deparaffinized and rehydrated. Then, antigen retrieval and quenching were performed in citric acid (10 $\mathrm{mM} \mathrm{pH6)}$ and $3 \%$ hydrogen peroxide, respectively. Immunohistochemistry for NOVA2, CD31, and HIF1 $\alpha$ was performed according to the instructions given in the R\&D Staining kit (HRP-DAB System, R\&D Systems, Minneapolis, MN, USA). As a negative control, 1 section for each slide was left without primary antibody. The primary antibodies were: anti-NOVA2 (C-16, Santa Cruz Biotechnology, Santa Cruz, CA, USA; dilution 1:50), anti-CD31 (Abcam, Cambridge, UK; dilution 1:25), and anti-HIF $1 \alpha(\mathrm{H} 1 \alpha 67$, Novus Biologicals, Littleton, CO, USA; dilution 1:10). The selected anti-CD31 and anti-HIF1 $\alpha$ antibodies have been previously described to positively stain ECs and tumor cells, respectively, in CRC tissues. ${ }^{9,13}$

NOVA2 expression was quantified by 2 operators using ImageJ (National Institutes of Health, Bethesda, MD, USA) with the Color Deconvolution Plugin. In order to avoid experimental variability between cases, and given that the basal expression of NOVA2 in human colon tissue vessels was uncharacterized, for each patient, we quantified NOVA2 expression in ECs of all the vessels of tumor and adjacent normal mucosa. For each section, the vessels with the same area and the same number of ECs in paired normal mucosa and tumor tissue were analyzed and compared. Then, the measurements were expressed as fold change of integrated optical density of tumoral vs normal ECs of vessels located in the same section. HIF $1 \alpha$ intensity was semi-quantified by 2 operators using Histoscore as follows: Histoscore $=1 \times(\%$ weak staining $)+2 \times(\%$ moderate staining $)+3 \times(\%$ strong staining $)$, which ranged from 0 (no nuclear and/or cytoplasmic staining 
detectable) to 300 (maximum staining intensity), as previously described. ${ }^{14}$ Considering that the existence of samples with no staining could be due to HIF $1 \alpha$ degradation because of tissue manipulation in normoxic conditions, ${ }^{8,15,16}$ we used only samples with Histoscore $>0$ ( $76 \%$ of the cases).

\section{In vitro endothelial model}

HUVECs (Thermo Fisher Scientific, Rockford, IL, USA) were used from passage 3 to passage 7 as previously described. ${ }^{17}$ For hypoxia experiment, HUVECs were cultured in both normoxic and hypoxic conditions $\left(1.5 \% \mathrm{O}_{2}\right.$ in the hypoxic chamber Xvivo System X3, Biospherix Ltd., Parish, NY, USA).

Total RNA was obtained as previously described. ${ }^{18}$ Two hundred nanogram of RNA were retrotranscribed and amplified with the Express 1step SS qRT-PCR Universal (Thermo Fisher Scientific). The gene expression analysis was performed according to the "Delta delta Ct Method" normalizing to the POLR $2 A$ gene as the housekeeping gene. Taqman assays (Thermo Fisher Scientific) used were: Hs01547115_m1 (for NOVA2), Hs99999905_m1 (for GAPDH), and Hs00172187_ m1 (for POLR2A).

Western blot was performed as previously described. ${ }^{19}$ Ten microgram and $35 \mu \mathrm{g}$ of proteins were used for NOVA2 and HIF $1 \alpha$ analysis, respectively. The following primary antibodies were used: anti-NOVA2 (SigmaAldrich, St Louis, MO, USA; 1:250), anti-ACTIN (AC-40, Sigma-Aldrich; 1:1,000), and anti-HIF $1 \alpha(\mathrm{H} 1 \alpha 67$, Novus Biologicals; 1:500).

\section{Statistical analysis}

Statistical analyses were performed with the GraphPad Prism version 6.00 (GraphPad Software, La Jolla CA, USA). Values were expressed as mean $\pm \mathrm{SD}$ if distribution was normal or as median (interquartile range) if nonnormal distribution was assessed. Normal distribution was analyzed using the D'Agostino and Pearson omnibus normality test. The Mann-Whitney $U$ test was used to compare NOVA2 data. Confidence level was fixed at 0.05 .

\section{Ethics approval and informed consent}

The study was approved by the Institutional Review Board of the University of Ferrara and conforms to the Declaration of Helsinki and the Guidelines for Good Clinical Practice.

\section{Results}

\section{NOVA2 upregulation in CRC vasculature}

As shown in Figure $1 \mathrm{~A}$ and $\mathrm{B}$, immunohistochemical analyses showed that NOVA2-positive cells were also
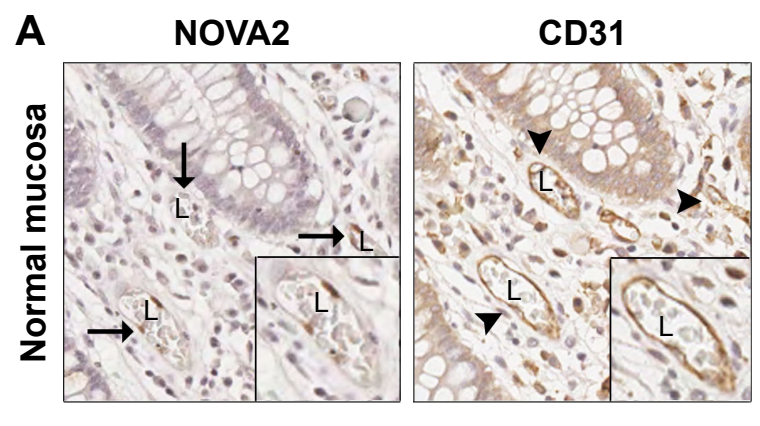

B
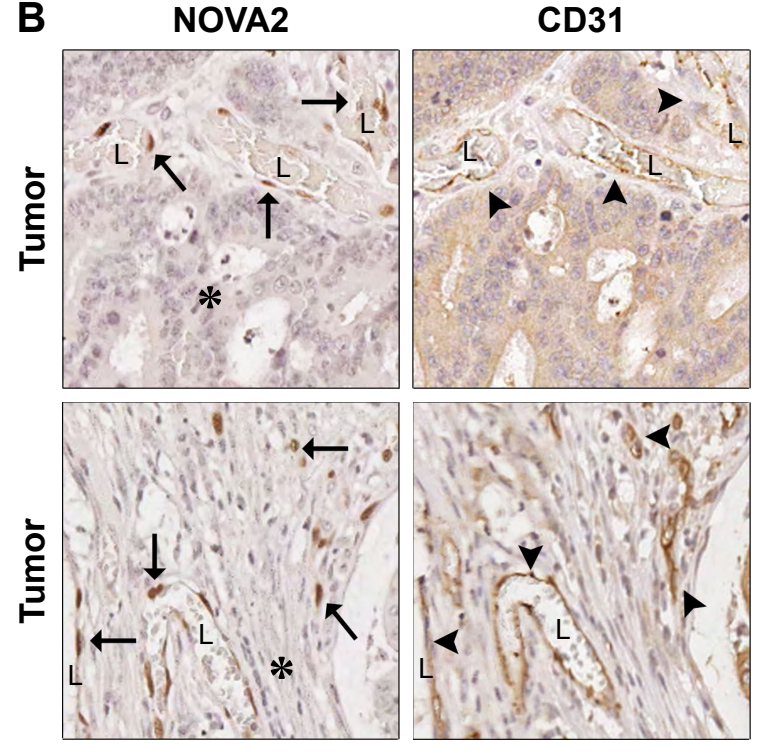

Figure I NOVA2 is expressed in ECs of human CRC.

Notes: NOVA2 and CD31 immunohistochemistry analysis in ECs of colon normal mucosa (A) and tumor tissue (B) in human CRC. Arrows indicate representative NOVA2 nuclear staining in ECs and arrowheads indicate representative membranous CD3I staining in ECs. "L" indicates lumen of the vessels. In (A), the insets show magnified images of the vessel indicated by the arrow at left. In (B), asterisks indicate tumor cells in the upper panel and stromal cells in the bottom panel. Magnification 200x.

Abbreviations: CRC, colorectal cancer; EC, endothelial cell.

positive for the endothelial marker CD31 in both normal mucosa and tumor tissue, confirming the endothelial nature of NOVA2. Conversely, non-ECs, such as tumoral and stromal cells, were negative for NOVA2 staining (Figure 1B).

To compare NOVA2 expression in the vasculature of normal and tumor tissues, the intensity of NOVA2 staining was quantitatively measured in vessels present in paired adjacent normal mucosa and tumor tissue for each patient. All vessels with the same area and amount of ECs were compared. As shown in Figure 2A and B, NOVA2 was significantly upregulated in tumor ECs compared to normal mucosa ECs (fold change=3.45; $P<0.001$ ). Conversely, in the sample from the additional patient who underwent neoadjuvant chemo- and radiotherapy, NOVA2 was downregulated in tumor ECs compared to the paired normal ECs (fold change $=0.82$ ). 

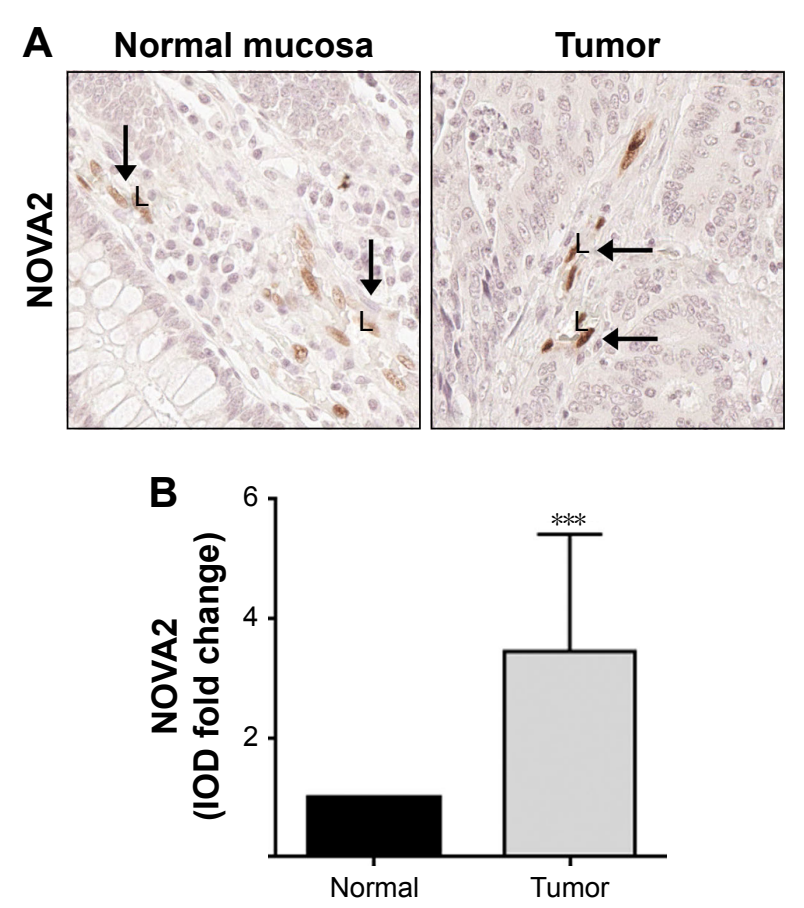

Figure 2 NOVA2 is upregulated in ECs of human CRC.

Notes: (A) Representative images of NOVA2 staining in ECs of paired normal mucosa and tumor tissue in human CRC. Arrows indicate NOVA2 nuclear staining in ECs. "L" indicates lumen of the vessels. Magnification 200x. (B) Quantification of NOVA2 staining in ECs of paired normal mucosa, set at $I$, and tumor tissue ( $n=40$ samples). Results are reported as mean $\pm S D$. $* * * P<0.001$ vs normal.

Abbreviations: CRC, colorectal cancer; EC, endothelial cell.

The analysis of correlations between NOVA2 and clinicopathological features of patients, such as tumor location, type, grade, and TNM stage, did not reveal statistically significant results mainly due to the low size of specific subgroups, although the analysis indicated a tendency of NOVA2 level in increasing in N1 stage tumors, in M1 stage tumors, and in MSS tumors (Figure S1).

\section{NOVA2 induction by hypoxia}

In order to gain insights into the mechanisms underlying the regulation of NOVA2 expression, and knowing that hypoxia regulates several genes involved in ECs function, ${ }^{8,10}$ the association between NOVA2 and the hypoxic marker HIF $1 \alpha$ was investigated in tumor tissue. For this purpose, we analyzed only the MSS samples $(n=33)$, since high MSI is demonstrated to affect the angiogenesis pathways. ${ }^{20,21}$ As shown in Figure 3A, low HIF1 $\alpha$ levels in the tumor corresponded to low NOVA2 intensity in ECs, while high HIF $1 \alpha$ levels corresponded to strong NOVA2 intensity in ECs. To evaluate the association between HIF $1 \alpha$ and NOVA2 levels, we semi-quantitatively analyzed HIF $1 \alpha$ staining and stratified the levels in 2 groups according to the median value (Histoscore=119). As shown in Figure 3B, tumors with low hypoxia (HIF1 $\alpha$ Histoscore $<119)$ were characterized by low NOVA2 levels, while tumors with high
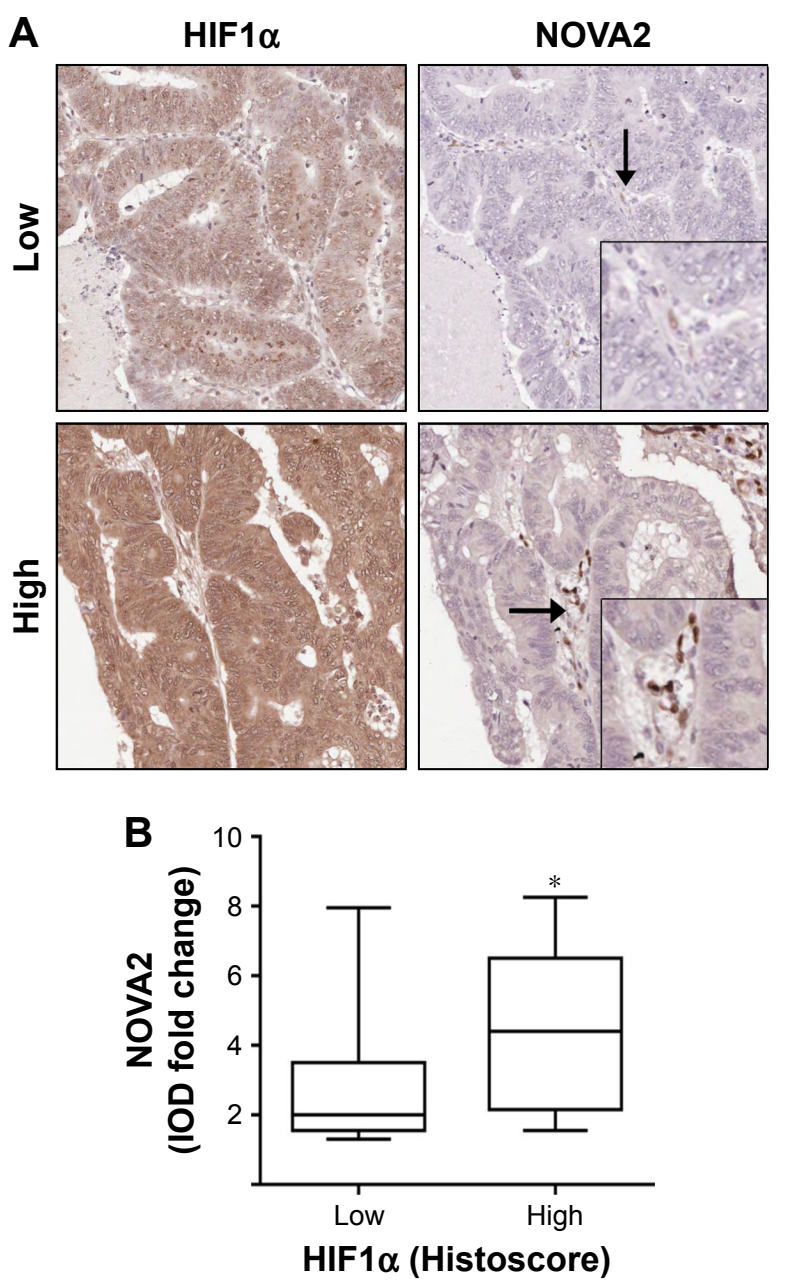

Figure 3 NOVA2 levels parallel HIFI $\alpha$ level in MSS tumors.

Notes: (A) Representative immunohistochemical images of low and high HIFI $\alpha$ and NOVA2 of the MSS tumors. Magnification 100x. The insets show magnified images of the vessels indicated by the arrows $(200 \times)$. (B) Association between levels of NOVA2 (expressed as IOD fold change of tumor tissue vs normal mucosa) and $\mathrm{HIFI} \alpha$ (expressed as Histoscore). Results are reported as median (IQR). Low$H I F I \alpha$ : Histoscore $<$ I 19. High-HIFI $\alpha$ : Histoscore $\geq$ I I9. $* P<0.05$.

Abbreviations: IOD, integrated optical density; IQR, interquartile range; MSS, microsatellite stable.

hypoxia (HIF $1 \alpha$ Histoscore $\geq 119$ ) resulted in high NOVA2 levels $(P=0.045)$.

Finally, we investigated NOVA2 expression in HUVEC in response to hypoxia. The experimental induction of hypoxia was verified at the protein level by the upregulation of HIF $1 \alpha$ (Figure 4A, left panel). In addition, hypoxia induction was confirmed also at mRNA level by the upregulation of the expression of $G A P D H$ mRNA $(P=0.036)$, another known marker of hypoxia ${ }^{22}$ (Figure 4A, right panel). As shown in Figure 4B, hypoxia induced the upregulation of NOVA2 protein (left panel) with a significant increase of NOVA2 mRNA expression $(P=0.029)$ (right panel).

\section{Discussion}

This is the first report demonstrating that NOVA2 is expressed in the vasculature of human CRC and, importantly, 
A

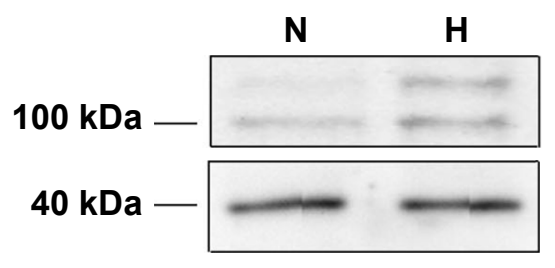

HIF1 $\alpha$

ACTIN

B

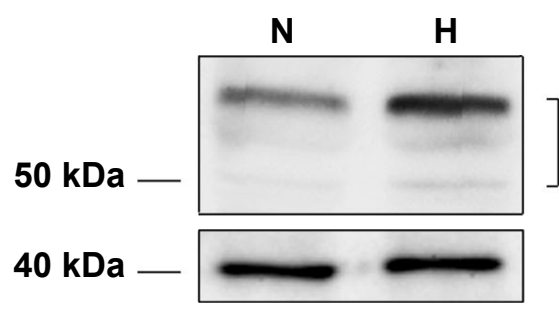

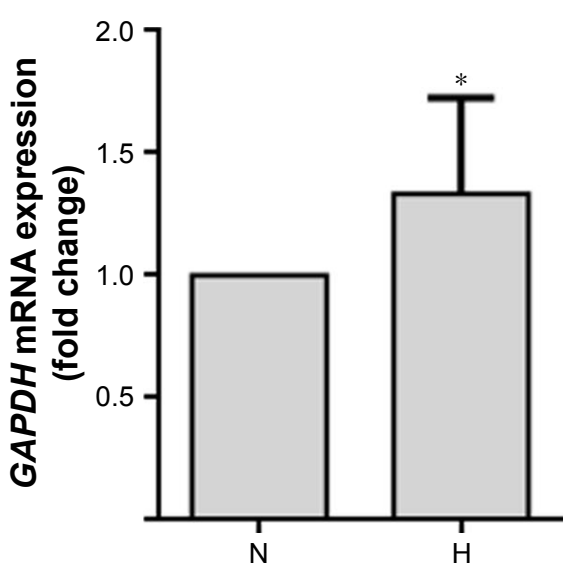

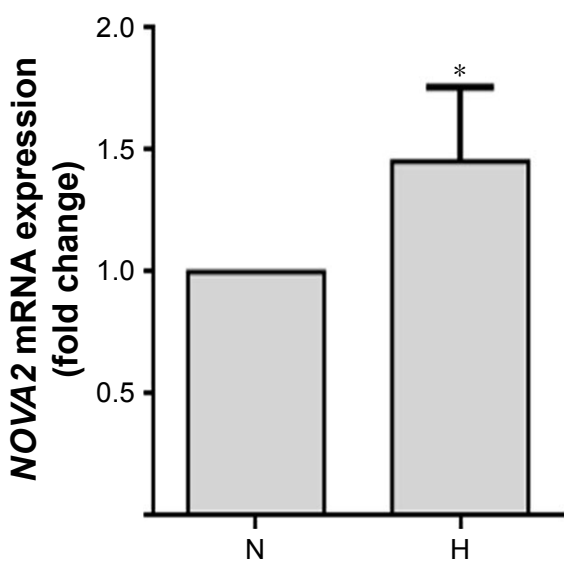

Figure 4 Hypoxia upregulates NOVA2 expression in vitro in ECs.

Notes: HUVECs were grown in normoxic $(N)$ or hypoxic $(H)$ conditions for 48 hours $(n=3)$. $(A)$ Left panel: representative Western blot of HIFI $\alpha$ and the loading control ACTIN. Right panel: quantitative reverse transcriptase (qRT)-PCR analysis of GAPDH mRNA levels expressed as fold change with respect to normoxic control set at I. Results are reported as mean \pm SD of 3 independent experiments. (B) Left panel: Western blot analysis of NOVA2 protein levels. ACTIN staining is shown as loading control. Representative blot of 3 independent experiments. Right panel: NOVA2 mRNA expression analyzed by qRT-PCR and expressed as fold change with respect to normoxic control set at I. Results are reported as mean \pm SD of three independent experiments. $* P<0.05$ vs normoxia.

that NOVA2 is significantly upregulated in tumor ECs. Moreover, high NOVA2 levels are associated with high HIF $1 \alpha$ levels, a marker of tumor hypoxia, ${ }^{9}$ both in CRC tissue and in an in vitro endothelial model.

The cohort of samples used for this pilot study was selected in order to include a variety of tumors types. A preliminary evaluation showed that NOVA2 median value tended to be higher in M1 and N1 stages tumors and in MSS tumors, although the low number of cases certainly did not allow the reaching of statistical significance. Interestingly, the analysis of 1 additional patient, who underwent neoadjuvant chemo- and radiotherapy, showed NOVA2 downregulation in tumor ECs, preliminarily suggesting an effect of therapy on NOVA2 expression.

Hypoxia is a key triggering factor of angiogenesis, which leads to transcriptional activation of hundreds of genes involved in vascular sprouting. ${ }^{10}$ Moreover, it is known that hypoxia regulates AS pathways in ECs and non-ECs. ${ }^{23-25}$ Of note, in the current study, we found that NOVA2 was upregulated by hypoxia, providing a new regulatory agent to study the effects of hypoxia on ECs based on AS modulation. NOVA 2 correlation with HIF $1 \alpha$ was investigated only in the MSS tumors because it is known that, despite high HIF $1 \alpha$ levels, MSI-H tumors paradoxically show low levels of VEGF and other angiogenic factors, ${ }^{20,21}$ suggesting that the high mutational rate may affect the typical angiogenic response to hypoxia.

As is known, AS factors overexpressed in tumors generate tumor-specific isoforms that contribute to the malignant phenotype and are potential tumor antigens. ${ }^{12}$ NOVA2 belongs to the family of NOVA proteins, initially identified as paraneoplastic disease antigens, ${ }^{3}$ and NOVA1, highly related to NOVA2, has been described to be expressed in different tumor types. ${ }^{7}$ Differently from NOVA1, we found NOVA2 overexpression not in tumor cells but rather in tumor vessels. Therefore, NOVA2 overexpression in ECs, through AS modulation of its target genes, might confer a pathogenic phenotype or generate new tumor-specific biomarkers and 
therapeutic targets at the tumor vasculature level with foremost clinical relevance. An example of aberrant AS in the clinical application is provided by the EDB antigen, a protein domain generated by AS in the FIBRONECTIN1 transcript only in tumor ECs, ${ }^{26}$ and targeted by the mAb L19 conjugated with therapeutic molecules currently investigated in preclinical and clinical studies. ${ }^{27-30}$ Thus, the identification of the NOVA2-modulated genes is noteworthy since it may contribute to the development of antiangiogenic drugs that could overcome the flaws of the existing therapies. Indeed, the benefit from the addition of antiangiogenic drugs to chemotherapy is currently limited, mainly because of the onset of drug resistance mechanisms. ${ }^{11,31}$

\section{Conclusion}

In conclusion, we report for the first time NOVA2 upregulation in the tumor vasculature and its direct association with hypoxia. Although we are aware of the limitations of this study, mainly due to the low number of specific subcases, our findings represent the proof of concept for future investigations in larger cohorts and different types of tumor that might allow the discovery of molecular mechanisms that link specific NOVA2 pathways to the reliance of tumor growth from neoangiogenesis.

\section{Data sharing statement}

Data will be shared by authors upon request.

\section{Acknowledgments}

We acknowledge Professor Giovanni Lanza and Doctor Elisabetta Khun for their scientific support and critical discussion of the manuscript. This work was supported by grants from Associazione Italiana per la Ricerca sul Cancro (AIRC, project 18055, fellowship to SG).

\section{Author contributions}

SG performed in vitro experiments and acquired the data; MVA performed tissues experiments and acquired the data; VT discussed the results; RP and LP performed hypoxia experiments and discussed the results; CB, CVF, and RG provided tissues samples and the clinical data and discussed the results; PS organized the study and discussed the results. All authors contributed toward data analysis, drafting and critically revising the paper, gave final approval of the version to be published, and agree to be accountable for all aspects of the work.

\section{Disclosure}

The authors report no conflicts of interest in this work.

\section{References}

1. Baralle FE, Giudice J. Alternative splicing as a regulator of development and tissue identity. Nat Rev Mol Cell Biol. 2017;18(7):437-451.

2. Ule J, Ule A, Spencer J, et al. Nova regulates brain-specific splicing to shape the synapse. Nat Genet. 2005;37(8):844-852.

3. Yang YY, Yin GL, Darnell RB. The neuronal RNA-binding protein Nova-2 is implicated as the autoantigen targeted in POMA patients with dementia. Proc Natl Acad Sci U S A. 1998;95(22):13254-13259.

4. Giampietro C, Deflorian G, Gallo S, et al. The alternative splicing factor Nova2 regulates vascular development and lumen formation. Nat Commun. 2015;6:8479.

5. Juan-Mateu J, Rech TH, Villate O, et al. Neuron-enriched RNA-binding proteins regulate pancreatic $\beta$ cell function and survival. J Biol Chem. 2017;292(8):3466-3480.

6. Vernia S, Edwards YJ, Han MS, et al. An alternative splicing program promotes adipose tissue thermogenesis. Elife. 2016;5:e17672.

7. Xin Y, Li Z, Zheng H, Ho J, Chan MTV, Wu WKK. Neuro-oncological ventral antigen 1 (NOVA1): implications in neurological diseases and cancers. Cell Prolif. 2017;50(4):e12348.

8. Cockman ME, Masson N, Mole DR, et al. Hypoxia inducible factor- $\alpha$ binding and ubiquitylation by the von Hippel-Lindau tumor suppressor protein. J Biol Chem. 2000;275(33):25733-25741.

9. Kuwai T, Kitadai Y, Tanaka S, et al. Expression of hypoxia-inducible factor- $1 \alpha$ is associated with tumor vascularization in human colorectal carcinoma. Int J Cancer. 2003;105(2):176-181.

10. Rey S, Semenza GL. Hypoxia-inducible factor-1-dependent mechanisms of vascularization and vascular remodelling. Cardiovasc Res. 2010;86(2):236-242.

11. Welti J, Loges S, Dimmeler S, Carmeliet P. Recent molecular discoveries in angiogenesis and antiangiogenic therapies in cancer. $J$ Clin Invest. 2013;123(8):3190-3200.

12. Bonomi S, Gallo S, Catillo M, Pignataro D, Biamonti G, Ghigna C. Oncogenic alternative splicing switches: role in cancer progression and prospects for therapy. Int J Cell Biol. 2013;2013:962038.

13. Katkoori VR, Manne U, Chaturvedi LS, et al. Functional consequence of the p53 codon 72 polymorphism in colorectal cancer. Oncotarget. 2017;8(44):76574-76586.

14. Bergadà L, Pallares J, Maria Vittoria $\mathrm{A}$, et al. Role of local bioactivation of vitamin D by CYP27A1 and CYP2R1 in the control of cell growth in normal endometrium and endometrial carcinoma. Lab Invest. 2014; 94(6):608-622.

15. Biswas S, Charlesworth PJ, Turner GD, et al. CD31 angiogenesis and combined expression of HIF- $1 \alpha$ and HIF- $2 \alpha$ are prognostic in primary clear-cell renal cell carcinoma (CC-RCC), but HIF $\alpha$ transcriptional products are not: implications for antiangiogenic trials and $\mathrm{HIF} \alpha$ biomarker studies in primary CC-RCC. Carcinogenesis. 2012; 33(9):1717-1725.

16. Evensen NA, Li Y, Kuscu C, et al. Hypoxia promotes colon cancer dissemination through up-regulation of cell migration-inducing protein (CEMIP). Oncotarget. 2015;6(24):20723-20739.

17. Voltan R, Zauli G, Rizzo P, et al. In vitro endothelial cell proliferation assay reveals distinct levels of proangiogenic cytokines characterizing sera of healthy subjects and of patients with heart failure. Mediators Inflamm. 2014;2014:257081.

18. Secchiero P, Voltan R, Rimondi E, et al. The $\gamma$-secretase inhibitors enhance the anti-leukemic activity of ibrutinib in B-CLL cells. Oncotarget. 2017;8(35):59235-59245.

19. Tisato V, Zauli G, Rimondi E, et al. Inhibitory effect of natural antiinflammatory compounds on cytokines released by chronic venous disease patient-derived endothelial cells. Mediators Inflamm. 2013; 2013:423407.

20. Inoue Y, Miki C, Watanabe H, Ojima E, Kusunoki M. Genomic instability and tissue expression of angiogenic growth factors in sporadic colorectal cancer. Surgery. 2006;139(3):305-311.

21. Furlan D, Sahnane N, Carnevali I, et al. Up-regulation of the hypoxiainducible factor-1 transcriptional pathway in colorectal carcinomas. Hum Pathol. 2008;39(10):1483-1494. 
22. Yamaji R, Fujita K, Takahashi S, et al. Hypoxia up-regulates glyceraldehyde-3-phosphate dehydrogenase in mouse brain capillary endothelial cells: involvement of $\mathrm{Na}+\mathrm{Ca} 2+$ exchanger. Biochim Biophys Acta. 2003;1593(2-3):269-276.

23. Weigand JE, Boeckel JN, Gellert P, Dimmeler S. Hypoxia-induced alternative splicing in endothelial cells. PLoS One. 2012;7(8):e42697.

24. Sena JA, Wang L, Heasley LE, Hu CJ, Cj H. Hypoxia regulates alternative splicing of HIF and non-HIF target genes. Mol Cancer Res. 2014; 12(9):1233-1243.

25. Brady LK, Wang H, Radens CM, et al. Transcriptome analysis of hypoxic cancer cells uncovers intron retention in EIF2B5 as a mechanism to inhibit translation. PLoS Biol. 2017;15(9):e2002623.

26. Castellani P, Borsi L, Carnemolla B, et al. Differentiation between highand low-grade astrocytoma using a human recombinant antibody to the extra domain-B of fibronectin. Am J Pathol. 2002;161(5):1695-1700.

27. Papadia F, Basso V, Patuzzo R, et al. Isolated limb perfusion with the tumor-targeting human monoclonal antibody-cytokine fusion protein L19-TNF plus melphalan and mild hyperthermia in patients with locally advanced extremity melanoma. J Surg Oncol. 2013;107(2):173-179.
28. Zegers CM, Rekers NH, Quaden DH, et al. Radiotherapy combined with the immunocytokine L19-IL2 provides long-lasting antitumor effects. Clin Cancer Res. 2015;21(5):1151-1160.

29. Palumbo A, Hauler F, Dziunycz P, et al. A chemically modified antibody mediates complete eradication of tumours by selective disruption of tumour blood vessels. Br J Cancer. 2011;104(7):1106-1115.

30. Menssen HD, Harnack U, Erben U, Neri D, Hirsch B, Dürkop H. Antibody-based delivery of tumor necrosis factor (L19-TNF $\alpha$ ) and interleukin-2 (L19-IL2) to tumor-associated blood vessels has potent immunological and anticancer activity in the syngeneic J558L BALB/c myeloma model. J Cancer Res Clin Oncol. 2018;144(3):499-507.

31. Kerr RS, Love S, Segelov E, et al. Adjuvant capecitabine plus bevacizumab versus capecitabine alone in patients with colorectal cancer (QUASAR 2): an open-label, randomised phase 3 trial. Lancet Oncol. 2016;17(11):1543-1557. 


\section{Supplementary material}
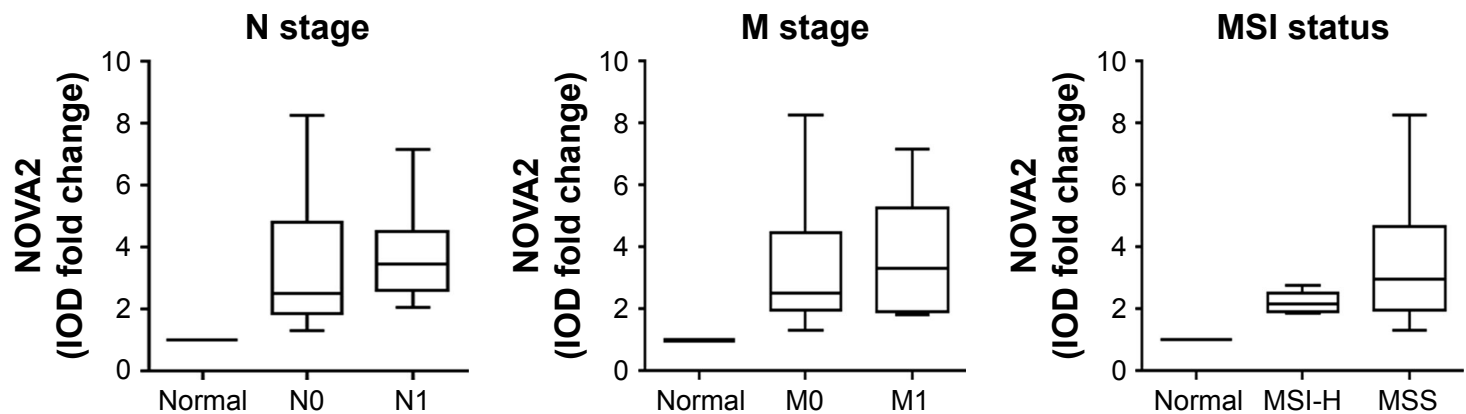

Figure SI NOVA2 levels are higher in NI, MI, and MSS tumors.

Notes: NOVA2 expression according to $\mathrm{N}$ stage, $M$ stage, and MSI status ( $n=40)$. Results are reported as median (IQR). N0: no regional lymph nodes metastasis. $\mathrm{NI}$ : metastasis in I-3 regional lymph nodes. M0: no distant metastasis. MI: distant metastasis.

Abbreviations: IQR, interquartile range; MSI-H, high microsatellite instability; MSS, microsatellite stable.

\section{Publish your work in this journal}

OncoTargets and Therapy is an international, peer-reviewed, open access journal focusing on the pathological basis of all cancers, potential targets for therapy and treatment protocols employed to improve the management of cancer patients. The journal also focuses on the impact of management programs and new therapeutic agents and protocols on

patient perspectives such as quality of life, adherence and satisfaction. The manuscript management system is completely online and includes a very quick and fair peer-review system, which is all easy to use. Visit http://www.dovepress.com/testimonials.php to read real quotes from published authors. 